\title{
Characteristics of differential lunar laser ranging
}

\author{
Mingyue Zhang ${ }^{1,2} \odot$, Jürgen Müller ${ }^{1} \odot$, Liliane Biskupek ${ }^{1} \odot$, and Vishwa Vijay Singh ${ }^{1,2} \oplus$ \\ ${ }^{1}$ Leibniz University Hannover, Institute of Geodesy (IfE), Schneiderberg 50, 30167 Hannover, Germany \\ e-mail: zhang@ife.uni-hannover.de \\ ${ }^{2}$ Institute for Satellite Geodesy and Inertial Sensing, German Aerospace Center (DLR), Callinstraße 36, 30167 Hannover, \\ Germany
}

Received 6 December 2021 / Accepted 2 February 2022

\begin{abstract}
Context. To obtain more details about the lunar interior, a station at Table Mountain Observatory of JPL will enable a new measurement of lunar laser ranging (LLR), known as differential lunar laser ranging (DLLR). It will provide a novel type of observable, namely, the lunar range difference, which is the difference of two consecutive ranges obtained via a single station swiftly switching between two or more lunar reflectors. This previously unavailable observation will have a very high level of accuracy (about $30 \mu \mathrm{m}$ ), mainly resulting from a reduction in the Earth's atmospheric error. In addition to the intended improvements for the lunar part, it is expected to contribute to improved relativity tests, for instance, the equivalence principle (EP).

Aims. This paper focuses on the simulation and investigation of the characteristics of DLLR.

Methods. Using simulated DLLR data, we analyzed and compared the parameter sensitivity, correlation, and accuracy obtained by DLLR with those attained by LLR.

Results. The DLLR measurement maintains almost the same sensitivity to certain parameters (called group A) as that of LLR, such as the lunar orientation parameters. For other parameters (called group B), such as station coordinates, it is shown to be less sensitive. However, owing to its extraordinary measurement accuracy, it not only retains nearly the same level of accuracy of group B as LLR, but it also improves the estimation of group A significantly (with the exception of reflector coordinates, due to the DLLR measuring mode). Also, DLLR increases the correlations among the reflectors and between stations and reflectors caused by its constellation. Additionally, we compared different switching intervals with respect to sensitivity and correlation. Large switching intervals are more beneficial for group B and the decorrelation of stations and reflectors. Furthermore, DLLR enhances the accuracy of EP tests.
\end{abstract}

Key words. Moon - astrometry - celestial mechanics - methods: data analysis

\section{Introduction}

Lunar laser ranging as an excellent space geodetic technique has provided the measurement of the distance between stations on the Earth and retro-reflectors on the Moon continuously for more than $50 \mathrm{yr}$. The five reflectors, deployed on the Moon since 1969, are all still working properly. The first corner cube retroreflector (CCR) array, namely, Apollo 11 (A11), was deployed on the Moon by American astronauts. Afterwards, Apollo 14 (A14), Apollo 15 (A15), Soviet Lunokhod 1 (L1), and Lunokhod 2 (L2) were placed on the Moon (Murphy 2013). In the future, there will be a new type of reflector with a different structure from the present reflector arrays and a great capability in terms of signal reflection (Ciocci et al. 2017; Turyshev et al. 2013; Garattini et al. 2013; Currie et al. 2011) meant to strengthen the space segment of LLR. The Earth segment of the IfE LLR data set includes six observatories. The Observatoire de la Côte d'Azur (OCA) in France has an overwhelming advantage in the amount of data collected. More than $60 \%$ of LLR data comes from this observatory. Moreover, in recent years, it successfully used infrared (IR) laser light to gain improved data distribution with respect to the reflectors and lunar phases (increasing the number of data at full Moon) (Courde et al. 2017). This is beneficial for the lunar orbital coverage and LLR data analysis. The Apache Point Observatory Lunar Laser-ranging Operation (APOLLO) in New Mexico, USA has the highest ranging accuracy among all stations. It is the only one which can achieve a few millimeters (about $8 \mathrm{~mm}$ in single shot), because of its larger aperture of the telescope (3.5 m) (Murphy et al. 2008). The McDonald observatory ${ }^{1}$ in Texas, USA is the only one equipped with three stations, namely, old McDonald, MLRS1, and MLRS2. The LLR data has also been obtained by other observatories: Matera Laser Ranging Observatory (MLRO) in Italy, Lunar Ranging Experiment (LURE) Observatory in Hawaii, USA and the Wettzell Laser Ranging System (WLRS) in Germany (Hofmann 2017). WLRS can also emit IR laser signals for ranging (Schreiber et al. 2019). With the improvements of the LLR stations, the main error sources of the LLR measurements are the reflectors on the Moon (Williams et al. 2009), the LLR model (Williams et al. 2004) and the Earth's atmosphere (which is the primary one). The atmosphere imposes a lower limit of $5 \mathrm{~mm}$ for the current LLR operations (Dehant et al. 2017).

With more than $50 \mathrm{yr}$ of tracking the Moon from Earth, LLR contributes to many scientific research fields related to the Earth-Moon system. For geodesy, LLR can be used to calculate the Earth orientation parameters (EOPs) that are related to the precession, nutation, rotation $\triangle U T 1$, and polar motion (Singh et al. 2021; Hofmann et al. 2018; Biskupek 2015). It can potentially provide a good verification for the estimation of $\Delta U T 1$ from very long baseline interferometry (VLBI). A joint analysis of EOPs is planned using these two techniques in the future.

\footnotetext{
1 The three stations of the McDonald Observatory are very close to each other. In our LLR and DLLR analysis, the three stations are linked by local ties and analyzed as one station for the process of the parameter estimation.
} 
Table 1. Comparison of LLR and DLLR.

\begin{tabular}{|c|c|c|c|c|c|c|c|}
\hline Technique & Laser & Constellation & $\begin{array}{c}\text { Station switching } \\
\text { (for one measurement) }\end{array}$ & Observation & Accuracy & Atmospheric error & Received photons \\
\hline LLR & Pulsed & $\begin{array}{l}\text { One station, } \\
\text { one reflector }\end{array}$ & No & Range & $\begin{array}{c}\text { About } 8 \mathrm{~mm} \\
\text { (LLR highest ranging accuracy, } \\
\text { for a single shot, } \\
\text { APOLLO station) }\end{array}$ & Major error source & $\begin{array}{l}\text { Single-photon level or a few photons } \\
\text { (one laser pulse) }\end{array}$ \\
\hline DLLR & $\mathrm{CW}$ & $\begin{array}{l}\text { One station, } \\
\text { two or more reflectors }\end{array}$ & Yes & Range difference & $\begin{array}{l}\text { About } 30 \mu \mathrm{m} \\
\text { (estimated according to } \\
\text { the error from the } \\
\text { Earth's atmosphere) }\end{array}$ & Significantly reduced & Large flux \\
\hline
\end{tabular}

For gravitational physics, LLR can test many parts of Einstein's theory of relativity, including the change of the gravitational constant, $G$, with time, the geodetic precession, the equivalence principle (EP) for the Earth and Moon in the gravitational field of the Sun, and of the galactic dark matter as well as the parametrized post-Newtonian (PPN) parameters, etc. (Biskupek et al. 2021; Zhang et al. 2020; Hofmann \& Müller 2018; Williams et al. 2012; Viswanathan et al. 2018). For selenophysics, LLR is sensitive to the lunar orbit, solid tides, and orientation (Müller et al. 2019; Williams et al. 2014). These quantities are all affected by the Moon's internal structure. Thus, LLR is a good tool to explore the properties of lunar interior, although there are still some uncertain issues of the deep part of the lunar interior, for example, the size, shape, and the rotation of the lunar core (Garcia et al. 2019).

Equipped with a 1-m telescope and a high-power continuous wave (CW) laser (operating at $1064 \mathrm{~nm}$ and amplitudemodulated with a linear chirp), a novel LLR station at Table Mountain Observatory (Dehant et al. 2017) of JPL will enable a new type of a geodesy technique known as Differential Lunar Laser Ranging (DLLR). A DLLR station can switch between two or more reflectors within a short time interval (nearly simultaneously). For each reflector, the station will receive the returned photons and track the phase of the returned modulated signal. It is expected that a DLLR station will receive a very large flux of photons which is the key factor to realize DLLR. Consequently, the square root of the number of received photons would no longer be a fundamental limitation to the LLR accuracy, rather, it will be the lack in knowledge in terms of the atmospheric delay. The phase measurement between one station and one reflector will contribute to the formation of the range measurement between them. The result of differencing any two consecutive range observations is one DLLR observable, that is, the range difference. The direct benefit of DLLR is unprecedented high-quality observations accurate to the level of about $30 \mu \mathrm{m}$. The main reason for this is that a large common part of the Earth's atmospheric error is reduced because of the similarity of the paths to the two reflectors which are measured successively. The angular width of the Moon viewed from the Earth is about 0.5 degree. This results in the differential atmospheric delay error of only about $31 \mu \mathrm{m}$ for two paths to the Moon. Due to the substantial decrease of the atmospheric error and the accurate DLLR data, it is anticipated that DLLR will provide a dramatic enhancement of our understanding of the deep lunar interior. This relates to, for instance, the boundary of the lunar core and mantle, the rotation of the core and the dissipation of a suspected partial melting area, and so on (Turyshev et al. 2018; Dehant et al. 2017). In addition, Turyshev et al. (2018) mentioned that DLLR can also be beneficial for some relativity tests, for example, related to the temporal variation of the gravitational constant $G$ and the EP.
This new LLR technique along with the new observable (with its excellent accuracy and performance) has motivated us to study its features, including its sensitivities to and correlations between as well as the accuracies of the estimated parameters (e.g., station and reflector coordinates, lunar orientation and rotation parameters, etc.). We simulated DLLR data and compared results based on it for various parameters to those of real LLR data. Apart from the investigation of the Newtonian parameters, such as reflector coordinates, we also preliminarily took a look at the effect of DLLR on the parameters related to the EP.

In Sect. 2, we compare the concept of LLR and DLLR. In Sect. 3, we provide the details on the simulation of DLLR data. The characteristics of DLLR are shown in Sect. 4. Section 5 presents our summary and outlook.

\section{Comparison of the LLR and DLLR concepts}

In this section, LLR and DLLR will be compared based on the aspects of the laser instrument, as well as the measurement process, intensity of the received signal, and accuracy of the observation. To highlight the differences between DLLR and LLR, a summary of their specific features is shown in Table 1. The information on DLLR in Table 1 is taken from Dehant et al. (2017).

\subsection{Laser}

The traditional laser used by all LLR stations to generate laser pulses is a pulsed laser. Pulsed lasers emit light pulses which are repeated at a fixed rate. Each pulse has a peak power and a pulse width which defines the time duration of this pulse. Even if the peak power is very high, a low average power for a time span is possible. In contrast, for CW lasers used in DLLR, the power is constant and continuous in time. The peak, minimum, and average power are almost identical.

\subsection{Measurement process}

Figure 1 shows the principle of an LLR measurement. It is a twoway link and for the uplink, one station on the Earth sends laser pulses to one reflector on the Moon. For the downlink, the reflector reflects the signal back to the station. The station receives the signal and determines the travel time for this round trip. Multiplied with the speed of light, we can get the distance between the station and the reflector.

As shown in Fig. 2, the measuring process of DLLR is more complex than that of LLR. At time $\mathrm{t}$, one station on the Earth sends amplitude-modulated signals generated by the $\mathrm{CW}$ laser to the first reflector. Then, it receives the reflected signal. Just as LLR, for this part of DLLR, it is still a two-way link. The range 


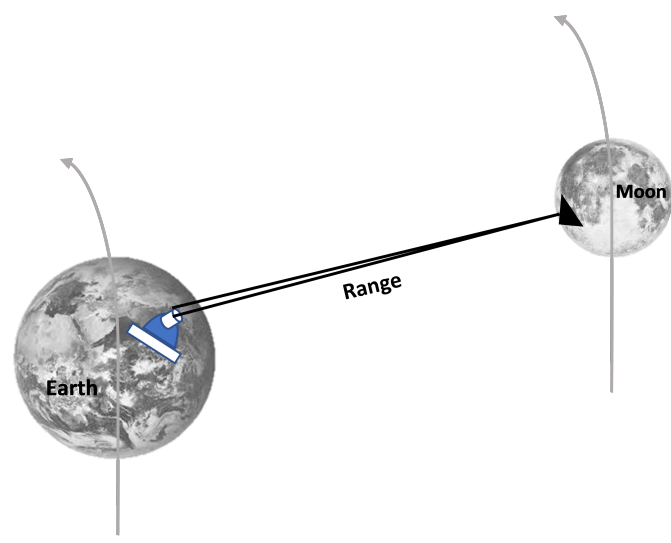

Fig. 1. Principle of an LLR measurement.

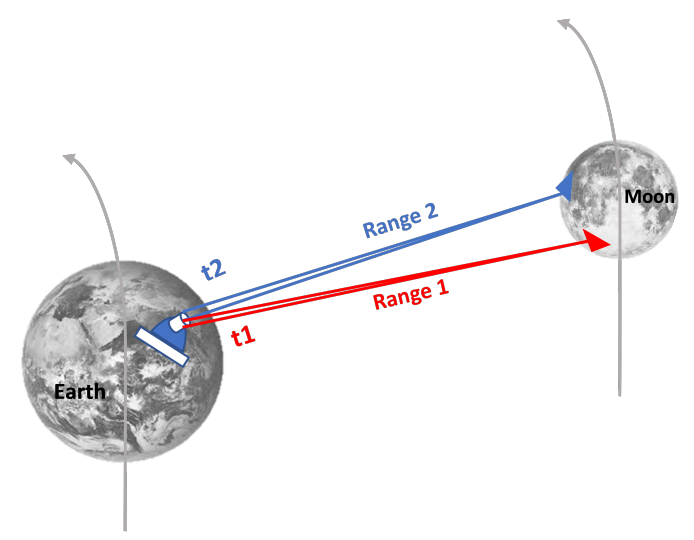

Fig. 2. Principle of a DLLR measurement.

between the station and reflector can be obtained from the phase difference of the outgoing and return signal. After a short interval, at time $t 2$, the same station will track another reflector and obtain another range. Differencing these two consecutive ranges, i.e., Range 2 minus Range1, we get the DLLR observable, the range difference. The switching time interval is the difference of $t 2$ and $t 1$.

\subsection{Signal intensity}

With a high-power CW laser, a large flux of received photons for DLLR is predicted. However, for an LLR station, the peak power of its pulsed laser can be very high where the average power is quite modest. Taking APOLLO as example, the average power is only about 2.3 W (Müller et al. 2019). In addition, there is a sizeable energy loss for the laser signal caused by instruments and signal paths. The primary reason is that laser pulses are affected by beam divergence resulting from the atmospheric turbulence and diffraction effects of the reflectors. Furthermore, paths of the transmitting and detection optics, as well as paths through atmosphere and the reflectivity of the retroreflectors can also cause signal loss. All these factors lead to the single-photon level. Less than or equal to one photon per laser pulse is received by most of the LLR stations (Murphy 2013; Chapront \& Francou 2006). Only the APOLLO station can conduct multi-photon detection, getting 3-5 photons per pulse (Dehant et al. 2017; Murphy et al. 2008).

\subsection{Observation accuracy}

The highest ranging accuracy of LLR is about $8 \mathrm{~mm}$ for a single shot achieved by APOLLO. The major reason for limiting the LLR accuracy is the Earth's atmosphere, with a lower limit of $5 \mathrm{~mm}$ (Dehant et al. 2017). For DLLR, the differential atmospheric delay error is about $30 \mu \mathrm{m}$. Thus, DLLR dramatically reduces the effect of the Earth's atmosphere and the accuracy of DLLR is about 200 times better than that of LLR (Turyshev et al. 2018).

\section{Simulation of DLLR data}

Because there is still no actual data available, we used the LLR simulation software by Hofmann (2017) and modified it for the simulation of DLLR data (Zhang 2022), namely, the range differences.

\subsection{General properties of our simulated DLLR data}

For a better and more fair comparison with LLR, the simulated DLLR data have the same number, distribution, and time span as those of the real LLR data ${ }^{2}$ we used. Additionally, the assumed stations and reflectors used in our DLLR simulation are the same as those of real LLR. The real LLR data provided by the LLR stations contain lots of information which includes the emitting epoch of the laser pulses, their travel time, and the accuracy of the measurement, meteorological data (pressure, temperature, and relative humidity), and the applied laser wavelength, the code of the station and the reflector, and so on (Hofmann 2017). One LLR measurement contains one station, one emitting epoch and one reflector. In contrast, one DLLR measurement includes one station, two emitting epochs ( $t 1$ and $t 2)$, and two different reflectors. For DLLR simulations, the station, the first epoch $t 1$ and the corresponding reflector are taken from the real LLR data. The second epoch $t 2$ is determined by adding the switching time interval (e.g., 1.5 or $15 \mathrm{~min}$ ) to $t 1$. In order to study the influence of the switching time interval on the DLLR observation, we used a small interval of $1.5 \mathrm{~min}$ (Slava G. Turyshev, 2021, personal commun.) and a relatively large interval, i.e., $15 \mathrm{~min}$ (Turyshev et al. 2018). The reflector for $t 2$ is any one of the remaining reflectors which are different from the reflector of $t 1$. According to the switching interval, we obtained two data groups, namely, the 1.5-min and 15-min group. Each has two data subgroups with different accuracies. One subgroup has a very low level of accuracy which is assumed to be equal to the ranging accuracy of standard LLR data to see what will happen for DLLR when the conditions of the measurement are not good. Another subgroup has an accuracy that is 200 times improved compared to standard LLR. Thus, we finally have four groups of DLLR data. Figure 3 shows the ranging accuracies of real LLR data we used, provided by eight LLR stations, namely, McDonald, MLRS1, MLRS2, APOLLO, OCA, LURE, MLRO, and WLRS, for five reflectors, namely, Apollo 11, Apollo 14, Apollo 15, Lunokhod 1, and Lunokhod 2, between 1970 and 2021. Table 2 shows the numbers and time spans of the real LLR data for the eight stations. There are some data gaps for LLR. For one period of time, not all stations conducted measurements which can clearly be seen in Fig. 3.

2 All LLR data used for this study are available from the Crustal Dynamics Data Information System (CDDIS) at NASA's Archive for Space Geodesy Data, USA, https://cddis.nasa.gov/Data and_Derived_Products/SLR/Lunar_laser_ranging_data.html (Noll 2010). 


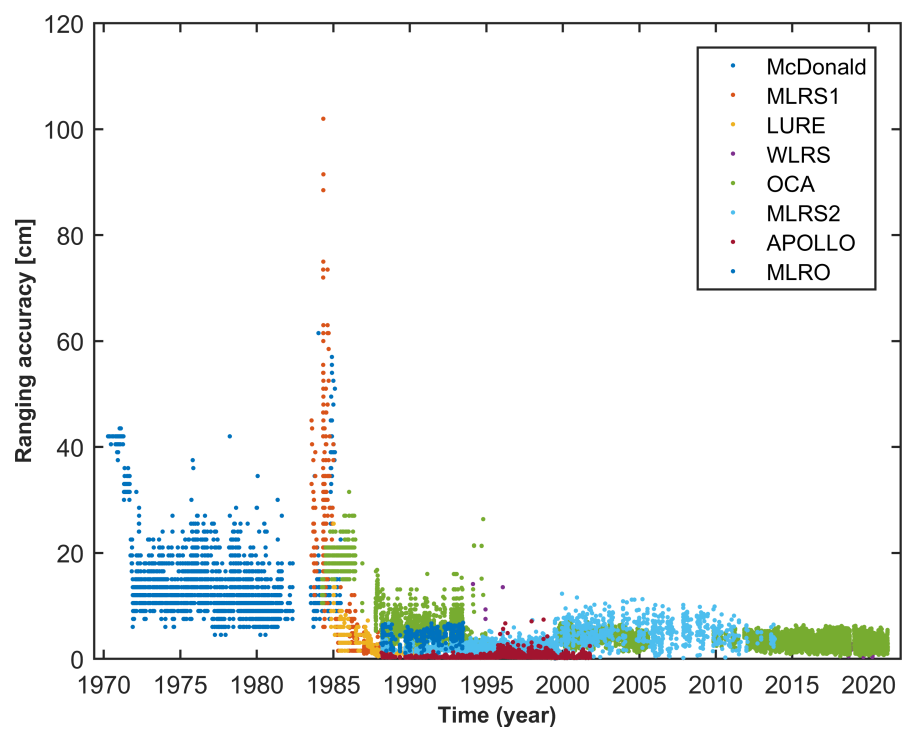

Fig. 3. Ranging accuracies of LLR stations.

Table 2. Numbers and time spans of LLR data.

\begin{tabular}{ccc}
\hline \hline Stations & Time span & Number of observations \\
\hline McDonald & $1970-1985$ & 3045 \\
MLRS1 & $1983-1988$ & 708 \\
MLRS2 & $1988-2013$ & 3131 \\
APOLLO & $2006-2016$ & 2584 \\
OCA & $1984-2021$ & 17445 \\
LURE & $1984-1990$ & 751 \\
WLRS & $1994-2020$ & 102 \\
MLRO & $2003-2021$ & 327 \\
All stations & $1970-2021$ & 28093 \\
\hline
\end{tabular}

The details of the model used for the DLLR simulation will be given in Sect. 3.2. For the model part related to the atmosphere, the meteorological data (pressure, temperature, and relative humidity), and wavelength of the station at $t 1$ and $t 2$ of one DLLR observation are required. They are taken from the real LLR data. As there are barely big changes in the atmospheric data within 1.5 or $15 \mathrm{~min}$, we kept the same meteorological data at $t 1$ and $t 2$ of a DLLR observation for our simulations. We also used the same wavelength for $t 1$ and $t 2$.

\subsection{Model of simulated DLLR data}

One DLLR measurement includes two ranging measurements from one station to two different reflectors on the Moon at two different emitting epochs, namely, $t 1$ and $t 2$. Assuming that the range of the uplink and downlink of the first ranging measurement are $\varrho_{\text {up } 1}$ and $\varrho_{\mathrm{dn} 1}$, the final range $\varrho_{1}$ is:

$\varrho_{1}=\frac{1}{2}\left(\varrho_{\mathrm{up} 1}+\varrho_{\mathrm{dn} 1}\right)$.

Similarly, for the second ranging measurement, the final range $\varrho_{2}$ is calculated from the uplink $\varrho_{\text {up2 }}$ and downlink $\varrho_{\mathrm{dn} 2}$ :

$\varrho_{2}=\frac{1}{2}\left(\varrho_{\mathrm{up} 2}+\varrho_{\mathrm{dn} 2}\right)$.
As a result, the DLLR range difference is

$$
\Delta \varrho=\varrho_{2}-\varrho_{1}+\varepsilon=\frac{1}{2}\left[\left(\varrho_{\mathrm{up} 2}-\varrho_{\mathrm{up} 1}\right)+\left(\varrho_{\mathrm{dn} 2}-\varrho_{\mathrm{dn} 1}\right)\right]+\varepsilon
$$

where $\varepsilon$ is white noise added according to the accuracy of DLLR.

All variables used in our LUNAR software (Hofmann 2017; Biskupek 2015) is in quadruple-precision floating-point format. According to the distance between the Earth and Moon (between 363000 and $406000 \mathrm{~km}$ ), this precision enables us to directly use the above formulation for simulating DLLR data which has a predicted accuracy of about $30 \mu \mathrm{m}$.

For the DLLR simulation, the most important part is the simulation of the range between one station and one reflector. The range model is the same as in our LLR analysis software LUNAR (Hofmann \& Müller 2018; Müller et al. 2014). Both LLR and DLLR, ranging from one station to one reflector, have one uplink and one downlink. For the uplink, the range model is:

$\varrho_{\text {up }}=\left|\boldsymbol{r} \boldsymbol{e} f_{\mathrm{B}}(t r)-\boldsymbol{s t a} \boldsymbol{a}_{\mathrm{B}}(t s e)\right|+\Delta \varrho_{r e l_{\mathrm{up}}}+\Delta \varrho_{a t m_{\mathrm{up}}}$.

For the downlink, the range model is:

$\varrho_{\mathrm{dn}}=\left|\boldsymbol{s t a} \boldsymbol{a}_{\mathrm{B}}(t s r)-\boldsymbol{r e} \boldsymbol{f}_{\mathrm{B}}(t r)\right|+\Delta \varrho_{r e l_{\mathrm{dn}}}+\Delta \varrho_{a t m_{\mathrm{dn}}}$,

where $s t a_{\mathrm{B}}(t s e), \boldsymbol{s t a} a_{\mathrm{B}}(t s r)$ are the positions of the station at the signal emitting time, $t s e$, and receiving time, $t s r$. In addition, $\boldsymbol{r} \boldsymbol{e} \boldsymbol{f}_{\mathrm{B}}(t r)$ is the position of the reflector at the time of signal reflection $t r$. The positions are considered in the Barycentric Celestial Reference System (BCRS). Here, $t s e, t s r$, and $t r$ are given in the Barycentric Dynamical Time (TDB; Biskupek 2015); $t$ se is initially given in the Coordinated Universal Time (UTC) which requires the transformation from UTC to TDB (Petit \& Luzum 2010).

The initial coordinates of a station and a reflector are in the Earth's and Moon's body-fixed frames, namely, the International Terrestrial Reference System (ITRS; Petit \& Luzum 2010) and Selenocentric Principal Axis System (PAS) (Park et al. 2021). The station coordinates have to be computed for the epochs of the observations ( $\left.s t a_{\mathrm{IR}}\right)$ with corrections resulting from the solid Earth tides, ocean tide loading, atmospheric loading, polar motion, and plate tectonics (Petit \& Luzum 2010). Then, $\boldsymbol{s t a}_{\mathrm{IR}}$ will be transformed into the coordinates in the Geocentric Celestial Reference System (GCRS), that is, $\boldsymbol{s t a}_{\mathrm{G}}$, by using the EOPs (precession, nutation, Earth rotation, and polar motion) and extra small rotation angles which are for the alignment of LLR and VLBI as the main technique for the estimation of EOPs. More details can be found in Biskupek (2015). For the reflector coordinates in PAS $\boldsymbol{r} \boldsymbol{e} \boldsymbol{f}_{\mathrm{P}}$, the degree- 2 tidal displacement is used for correcting them (Petit \& Luzum 2010) to get $\boldsymbol{r} \boldsymbol{e} \boldsymbol{f}_{\mathrm{PR}}$. Three Euler angles $\left(\varphi_{\mathrm{m}}, \theta_{\mathrm{m}}, \psi_{\mathrm{m}}\right)$ (Park et al. 2021) which parameterize the orientation of the lunar mantle are used to transform $\boldsymbol{r e} \boldsymbol{f}_{\mathrm{PR}}$ into the coordinates of the Selenocentric Celestial Reference System (SCRS) $\boldsymbol{r} \boldsymbol{e} \boldsymbol{f}_{\mathrm{S}}$ (Biskupek 2015).

The equations for converting the station coordinates in the Earth-centered GCRS $\boldsymbol{s t a}_{\mathrm{G}}$ and the reflector coordinates in the Moon-centered SCRS $\boldsymbol{r} \boldsymbol{e} \boldsymbol{f}_{\mathrm{S}}$ to the coordinates of BCRS $\boldsymbol{s} \boldsymbol{t} \boldsymbol{a}_{\mathrm{B}}$ and $\boldsymbol{r e} f_{\mathrm{B}}$ are:

$\boldsymbol{s t a} \boldsymbol{a}_{\mathrm{B}}=\boldsymbol{r}_{\mathrm{e}}+\boldsymbol{s} \boldsymbol{t a} \boldsymbol{a}_{\mathrm{G}}\left(1-\frac{U_{\mathrm{e}}}{c^{2}}-L_{\mathrm{C}}\right)-\frac{1}{2}\left(\frac{\boldsymbol{v}_{\mathrm{e}} \cdot \boldsymbol{s} \boldsymbol{t} \boldsymbol{a}_{\mathrm{G}}}{c^{2}}\right) \boldsymbol{v}_{\mathrm{e}}$,

$\boldsymbol{r} \boldsymbol{e} \boldsymbol{f}_{\mathrm{B}}=\boldsymbol{r}_{\mathrm{m}}+\boldsymbol{r} \boldsymbol{e} \boldsymbol{f}_{\mathrm{S}}\left(1-\frac{U_{\mathrm{m}}}{c^{2}}-L_{\mathrm{B}}\right)-\frac{1}{2}\left(\frac{\boldsymbol{v}_{\mathrm{m}} \cdot \boldsymbol{r} \boldsymbol{e} \boldsymbol{f}_{\mathrm{S}}}{c^{2}}\right) \boldsymbol{v}_{\mathrm{m}}$, 
with the position vector of the Earth, $\boldsymbol{r}_{\mathrm{e}}$, and the position vector of the Moon, $\boldsymbol{r}_{\mathrm{m}}$, in the BCRS. $U_{\mathrm{e}}$ and $U_{\mathrm{m}}$ are the gravitational potential of the Sun at the geocenter and the center of the Moon. We have: $L_{\mathrm{C}}=1.48082686741 \times 10^{-8} ; L_{\mathrm{B}}=1.550519768 \times$ $10^{-8} ; c$ is the speed of light. Together with $\boldsymbol{r}_{\mathrm{e}}$ and $\boldsymbol{r}_{\mathrm{m}}$, the velocity vector of the Earth, $\boldsymbol{v}_{\mathrm{e}}$, and the velocity vector of the Moon, $\boldsymbol{v}_{\mathrm{m}}$, in BCRS are numerically integrated by their equations of motion based on some initial positions and velocities (Park et al. 2021; Biskupek 2015; Petit \& Luzum 2010). The most important part of the equations of motion is the interaction of the bodies as point masses which are described by the Einstein-Infeld-Hoffmann (EIH) equations (Will 2018; Einstein et al. 1938). Furthermore, the interaction of the point masses with the non-spherical parts and the lunar acceleration caused by the tides of the Earth resulting from the Moon and Sun are also included (Hofmann \& Müller 2018; Müller et al. 2014; Park et al. 2021).

There are two kinds of range corrections included in Eqs. (4) and (5). The first kind $-\Delta \varrho_{r e l_{\text {up }}}$ for the uplink in the Eq. (4) and $\Delta \varrho_{r e l_{\mathrm{dn}}}$ for the downlink in the Eq. (5) - is caused by the Shapiro time delay (Moyer 1971; Shapiro 1964). The second one is due to the Earth's atmosphere ( $\Delta \varrho_{a t m}$ for the uplink in the Eq. (4) and $\Delta \varrho_{a t m_{\mathrm{dn}}}$ for the downlink in the Eq. (5), later referred to as $\mathrm{AR})$. The AR is related to the meteorological data of the station, namely, the pressure, $\mathrm{P}_{\mathrm{s}}$; temperature, $\mathrm{t}_{\mathrm{s}}$; relative humidity, hum; etc. (Mendes \& Pavlis 2004; Mendes et al. 2002). The model details can be found in Petit \& Luzum (2010).

The pressure, $\mathrm{P}_{\mathrm{s}}$, temperature, $\mathrm{t}_{\mathrm{s}}$, and relative humidity, hum, can change within 1.5 or $15 \mathrm{~min}$. This motivated us to investigate the effects of the change of $\mathrm{P}_{\mathrm{s}}, \mathrm{t}_{\mathrm{s}}$, and hum on DLLR. The difference between the AR for two epochs ( $t 1$ and $t 2$ ) of a DLLR measurement is part of the range difference caused by the atmospheric delay (later referred to as atmospheric range difference (ARD)). According to Eq. (1) and (2), for DLLR, the AR for $t 1$ or $t 2$ is calculated by half of the sum of the AR for the uplink and downlink. As reference, the same meteorological data at $t 1$ and $t 2$ are taken. Then three test cases consider the changes: one for $\mathrm{P}_{\mathrm{s}}$, one for $\mathrm{t}_{\mathrm{s}}$, and one for hum. The $\mathrm{P}_{\mathrm{s}}, \mathrm{t}_{\mathrm{s}}$, and hum values at the first epoch $t 1$ are the same as those of the reference. For the $\mathrm{P}_{\mathrm{s}}$ test case, the pressure at the second epoch $t 2$ is the pressure at $t 1$ plus $0.0025 \mathrm{hPa}^{3}$, but the $\mathrm{t}_{\mathrm{s}}$ and hum values are the same as those at $t 1$. For $\mathrm{t}_{\mathrm{s}}$, we kept the $\mathrm{P}_{\mathrm{s}}$ and hum values at $t 2$ the same as those at $t 1$ and added $0.05^{\circ} \mathrm{C}$ to the temperature at $t 1$ to get the temperature at $t 2$. The hum test case assumes no change of $\mathrm{P}_{\mathrm{s}}$ and $\mathrm{t}_{\mathrm{s}}$ at $t 1$ and $t 2$, but $0.25 \%$ was added to the relative humidity of time $t 1$ to get that of $t 2$. Furthermore, $t 2$ equals $t 1$ plus 1.5 or $15 \mathrm{~min}$. The effect of the change of $\mathrm{P}_{\mathrm{s}}, \mathrm{t}_{\mathrm{s}}$, and hum during a switching time interval of DLLR ( $t 2$ minus $t 1)$ can be calculated by the ARD from the $P_{s}, t_{s}$, and hum test cases minus the ARD from the reference, respectively. The effect of the change of $P_{s}$, $\mathrm{t}_{\mathrm{s}}$, and hum on DLLR with a 1.5 -min switching interval is different from that on DLLR with a 15 -min switching interval by just a few micrometers. The 15-min interval has a higher possibility to have meteorological changes than the 1.5-min interval. Therefore, taking the 15-min results, Figs. 4-6 show the impact of the changes of $\mathrm{P}_{\mathrm{s}}, \mathrm{t}_{\mathrm{s}}$, and hum on 15-min DLLR observations for 8 LLR stations. The effect of pressure, temperature, and relative humidity changes are within about $35 \mu \mathrm{m}, 30 \mu \mathrm{m}$, and $40 \mu \mathrm{m}$, respectively, as the pressure, temperature, and relative humidity only changed by $0.0025 \mathrm{hPa}, 0.05^{\circ} \mathrm{C}$, and $0.25 \%$ within $15 \mathrm{~min}$. Each of these effects is comparable with the envisaged accuracy of DLLR according to the Earth's atmosphere (about

\footnotetext{
$3 \mathrm{hPa}$ means hectopascal. It is the unit of pressure, equivalent to millibar. $1 \mathrm{hPa}=1 \mathrm{mbar}$.
}

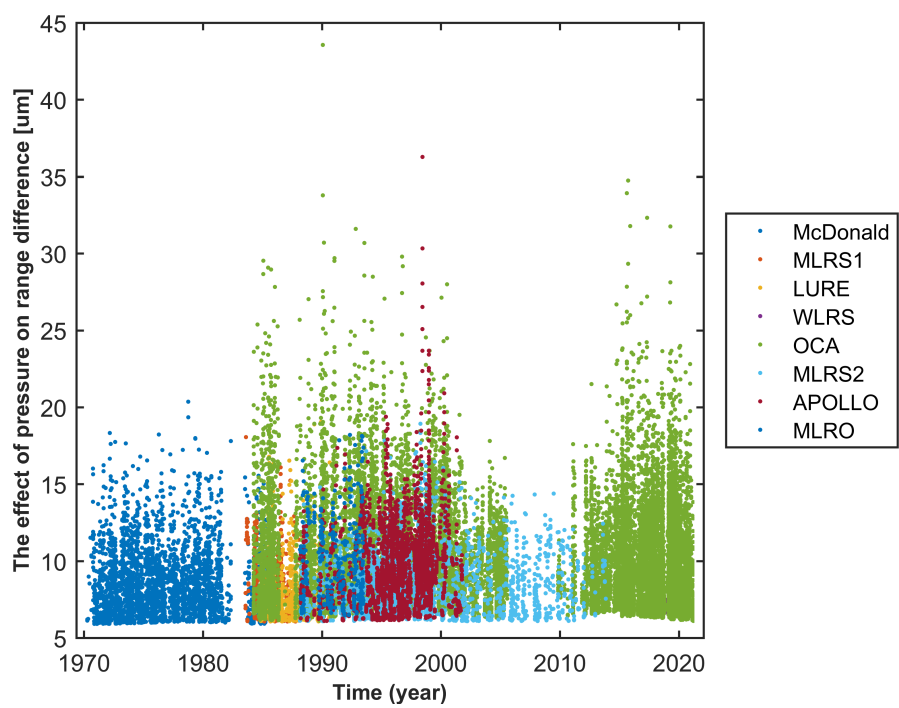

Fig. 4. Effect of pressure changes on DLLR observations for a 15-min switching interval.

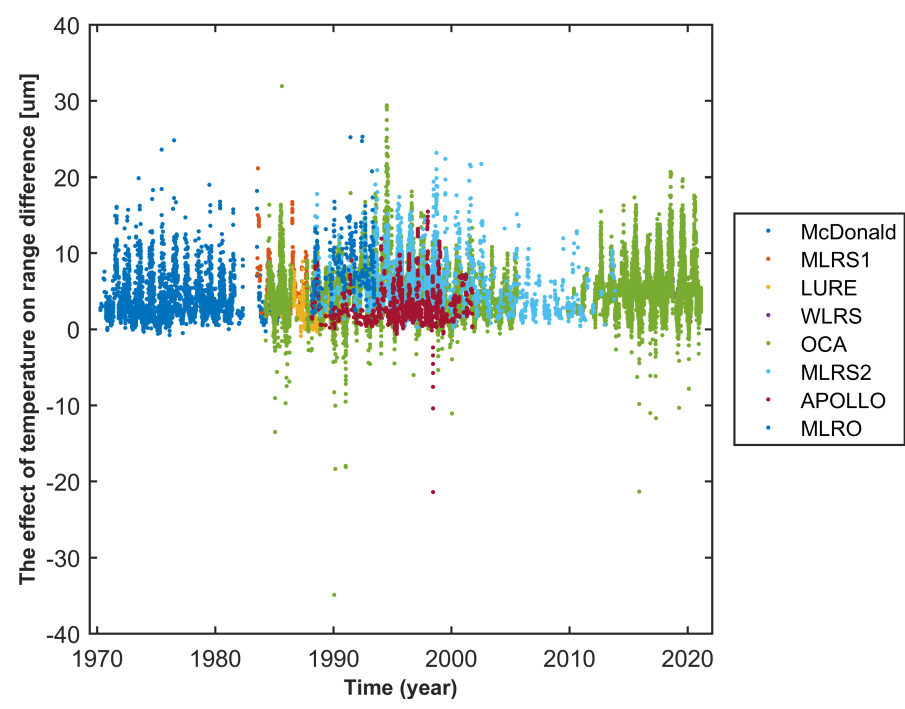

Fig. 5. Effect of temperature changes on DLLR observations for a 15min switching interval.

$30 \mu \mathrm{m})$. Thus, the meteorological changes during a short switching time interval may slightly affect the DLLR measurements. However, presently, according to real LLR data, the precision of the pressure, temperature, and relative humidity provided by LLR stations are about $0.05 \mathrm{hPa}, 0.1^{\circ} \mathrm{C}$, and $1 \%$ only. Thus, the collection of meteorological data at future DLLR stations has to be improved.

\section{DLLR characteristics}

As noted in Sect. 3.1, we simulated four DLLR data groups: two for a 1.5-min switching interval and two for a 15-min switching interval. The accuracy of one group for each time interval is the same as the ranging accuracy of LLR and the accuracy of the second group is 200 times better. Eventually, the accuracy of the DLLR data with a 1.5-min switching interval should be different from that with a 15-min switching interval. But, in this paper, we assumed the same accuracy for simplicity. The investigations of the characteristics of DLLR include the sensitivities to the 


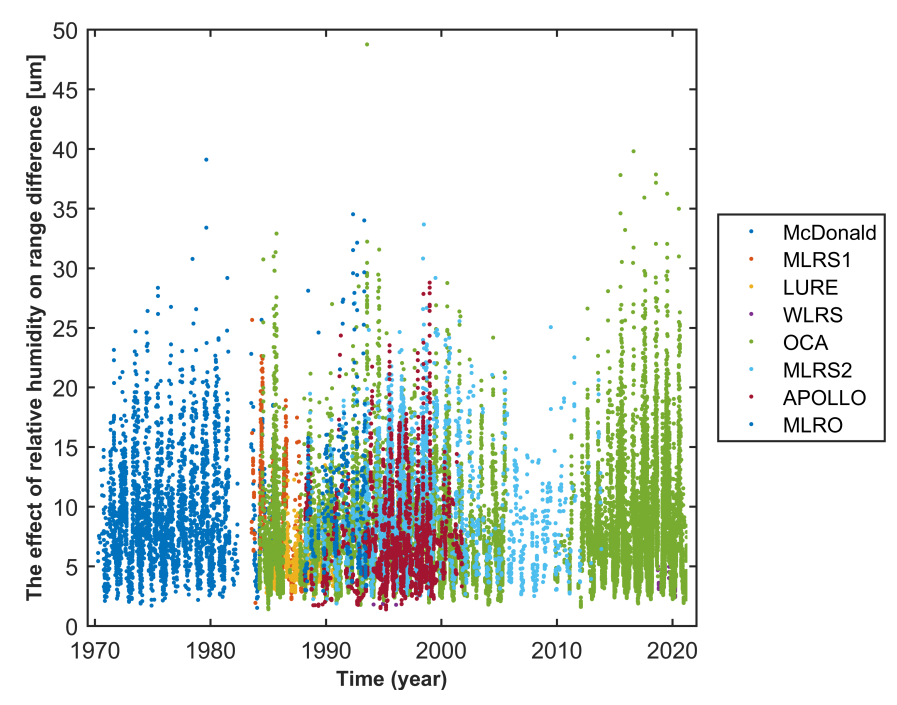

Fig. 6. Effect of relative humidity changes on DLLR observations for a 15-min switching interval.

estimated parameters, for example, the reflector coordinates, the orientation parameters of the lunar mantle, as well as the correlations between and the accuracies of the estimated parameters determined from DLLR compared to those from LLR. It should be noted that the orientation parameters of the lunar mantle, the angular velocities of the lunar mantle and core, and the 3D positions and velocities of the Moon involved in our analysis are calculated by numerical integration. Thus, the estimated parameters in DLLR and LLR are the initial orientation $\left(\varphi_{\mathrm{m} 0}, \theta_{\mathrm{m} 0}, \psi_{\mathrm{m} 0}\right)$, angular velocities $\left(\omega_{\mathrm{m} x 0}, \omega_{\mathrm{m} y 0}, \omega_{\mathrm{m} z 0}\right)$ of the lunar mantle, the initial angular velocities of the lunar core $\left(\omega_{\mathrm{c} x 0}, \omega_{\mathrm{c} y 0}, \omega_{\mathrm{c} z 0}\right)$, the initial 3D-positions $\left(X_{0}, Y_{0}, Z_{0}\right)$, and velocities $\left(V_{x 0}, V_{y 0}, V_{z 0}\right)$ of the Moon at Julian day (TDB) 2440400.5. For the correlations between the parameters, we focus on the correlations among the reflector coordinates themselves and between station and reflector coordinates (see Sect. 4.2). By comparing the sensitivities and correlations of the DLLR data group of low and high accuracy, we found that the sensitivities and correlations are nearly not related to the accuracy of the data used. Thus, the DLLR groups used for the investigation of the sensitivities and the correlations are those with high accuracy (200 times improvement compared to the present LLR ranging accuracy).

\subsection{Sensitivities to the analyzed parameters}

In this section, two comparisons related to the DLLR parameter sensitivities are analyzed. One comparison is between the DLLR data with a 1.5-min switching interval and the real LLR data. Another comparison is between the two kinds of simulated DLLR data, namely, DLLR data with 1.5-min and 15-min switching intervals. The sensitivity of LLR to a parameter is given by the partial derivative of the range between a station and a reflector, $\rho$, with respect to this parameter. For example, the sensitivity of LLR to one of the three initial Euler angles $\varphi_{\mathrm{m} 0}$ is:

$\frac{\partial \rho}{\partial \varphi_{\mathrm{m} 0}}=\sum_{i=1}^{3}\left(\frac{X m_{i}+X r e f_{i}-X s t a_{i}}{\rho} \cdot\left(\frac{\partial X m_{i}}{\partial \varphi_{\mathrm{m} 0}}+\frac{\partial X r e f_{i}}{\partial \varphi_{\mathrm{m} 0}}\right)\right)$,

where $X m_{i}, X r e f_{i}$, and $X s t a_{i}$ are the $i$ th components of the coordinates for the center of the Moon with respect to the geocenter, for the reflector with respect to the selenocenter, and for the station with respect to the geocenter. All the vectors are given in

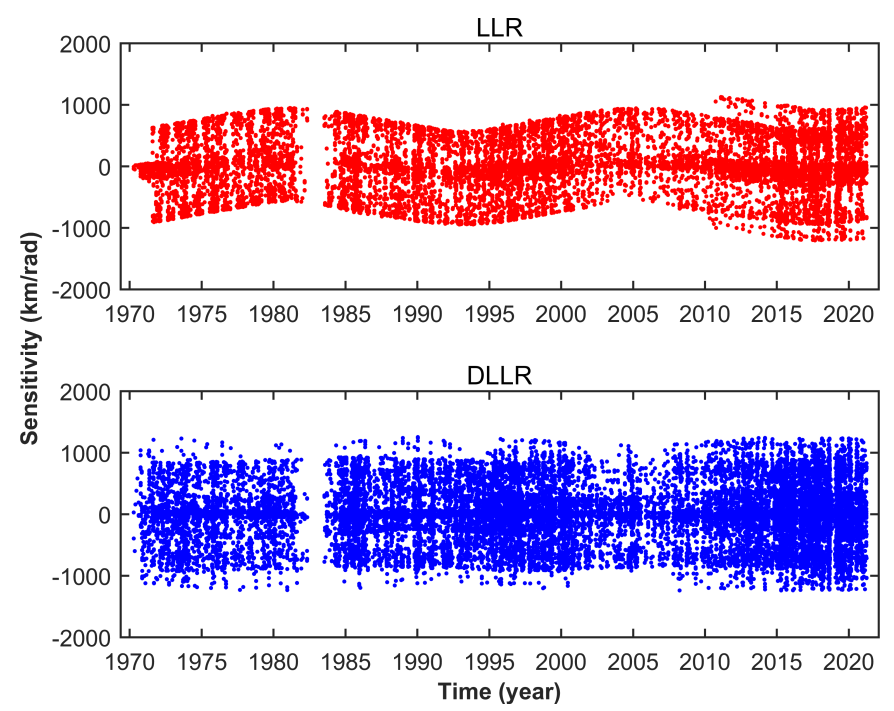

Fig. 7. Sensitivity to $\theta_{\mathrm{m} 0}$ for LLR (upper panel) and DLLR (lower panel).

the BCRS. The DLLR sensitivity to a parameter is the difference of the partial derivatives of two ranges (from one station to two different reflectors) with respect to this parameter. It should be noted that the two ranges of one DLLR measurement are for two different reflectors; thus, only one range is actually used for the calculation of the partial derivatives of the coordinates for a reflector.

\subsubsection{Comparison of the parameter sensitivities for DLLR and LLR}

Here, we compare the parameter sensitivity for the range difference (DLLR) and range (one-way, LLR). When comparing the values of the partial derivatives of the estimated parameters from LLR and DLLR, the parameters can be divided into two groups, namely: group A and B, where DLLR and LLR have almost the same sensitivities to the parameters of group A. Nearly all estimated parameters related to the lunar part belong to group A, including the $\left(\varphi_{\mathrm{m} 0}, \theta_{\mathrm{m} 0}, \psi_{\mathrm{m} 0}\right),\left(\omega_{\mathrm{m} x 0}, \omega_{\mathrm{m} y 0}, \omega_{\mathrm{m} z 0}\right),\left(\omega_{\mathrm{c} x} 0\right.$, $\left.\omega_{\mathrm{c} y 0}, \omega_{\mathrm{c} z 0}\right)$, low-degree gravitational coefficients of the lunar gravity field $\left(C_{22}, C_{33}\right.$, etc.), the oblateness of the lunar core $\left(f_{\text {core }}\right)$, and the friction coefficient between the core and mantle $(k v c)$, which is related to the dissipation of the core/mantle boundary, and so on. These parameters are relevant to the modelling of the lunar interior (Park et al. 2021; Williams \& Boggs 2008). Figures 7-9 show the DLLR and LLR sensitivities to $\theta_{\mathrm{m} 0}$, $C_{22}, f_{\text {core }}$ as examples. Reflector coordinates are also included in group A. From Figs. 10 and 11, we can see that DLLR and LLR are more sensitive to the $x$-coordinate than to the $y$ - and $z$ coordinates. Figure 10 shows the sensitivity to the $x$-coordinate of the A15 reflector $\left(X_{\text {ref_A15 }}\right)$. There are three parts (LLR and DLLR-parts 1 and 2) for a better comparison. DLLR contains a negative sensitivity part (DLLR-part 1) and positive sensitivity part (DLLR-part 2) for $X_{\text {ref_A15 }}$. The sensitivity to the $x$-coordinate from all DLLR data with a 1.5 -min switching interval is composed of the DLLR-parts 1 and 2. As DLLR and LLR have very similar sensitivities to the $y$ - and $z$-coordinates of A15 $\left(Y_{\text {ref_A15 }}, Z_{\text {ref_A15 }}\right)$, Fig. 11 just shows the DLLR sensitivity to $Y_{\text {ref_A15 }}$ and $Z_{\text {ref_A } A 15}$. The sensitivity to the $x$-coordinate is different from the sensitivity to the $y$ - and $z$-coordinates, because the $\mathrm{x}$-axis of the lunar body-fixed coordinate system points to the average direction of the Earth. 


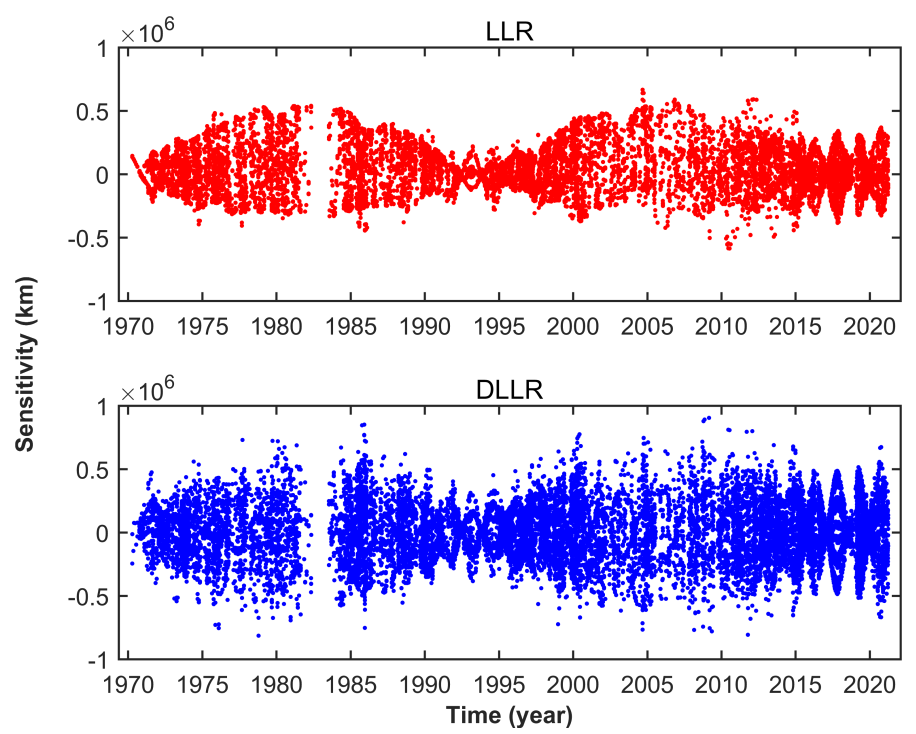

Fig. 8. Sensitivity to $C_{22}$ for LLR (upper panel) and DLLR (lower panel).

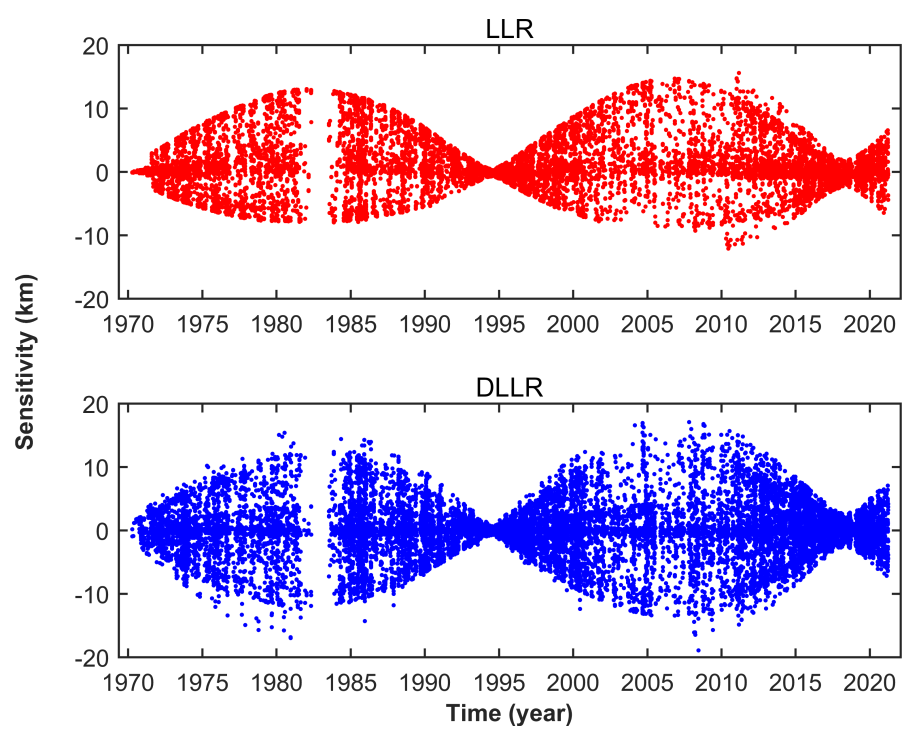

Fig. 9. Sensitivity to $f_{\text {core }}$ for LLR (upper panel) and DLLR (lower panel).

For the parameters of group B, DLLR becomes less sensitive than LLR. However, this disadvantage is compensated by the benefit from the high accuracy of DLLR observations, which are shown in Sect. 4.3. All the estimated parameters related to the stations are in group B, for instance, the station coordinates (see Fig. 12 for the $z$-component of OCA $\left(Z_{\text {sta_OCA }}\right)$ as an example), resulting from the cancelling effect of DLLR on the station. Moreover, for the initial 3D-positions $\left(X_{0}, Y_{0}, Z_{0}\right)$ and velocities $\left(V_{x 0}, V_{y 0}, V_{z 0}\right)$ of the Moon, DLLR also has less sensitivity (see Fig. 13 for $Z_{0}$ as an example).

\subsubsection{Comparison of the parameter sensitivities for DLLR with different switching intervals}

The sensitivities to the estimated parameters of the lunar part calculated by DLLR data with a 1.5-min switching interval are practically equal to those determined by DLLR data with a 15min switching interval (see Fig. 14 for an example based on $k v c$ ).

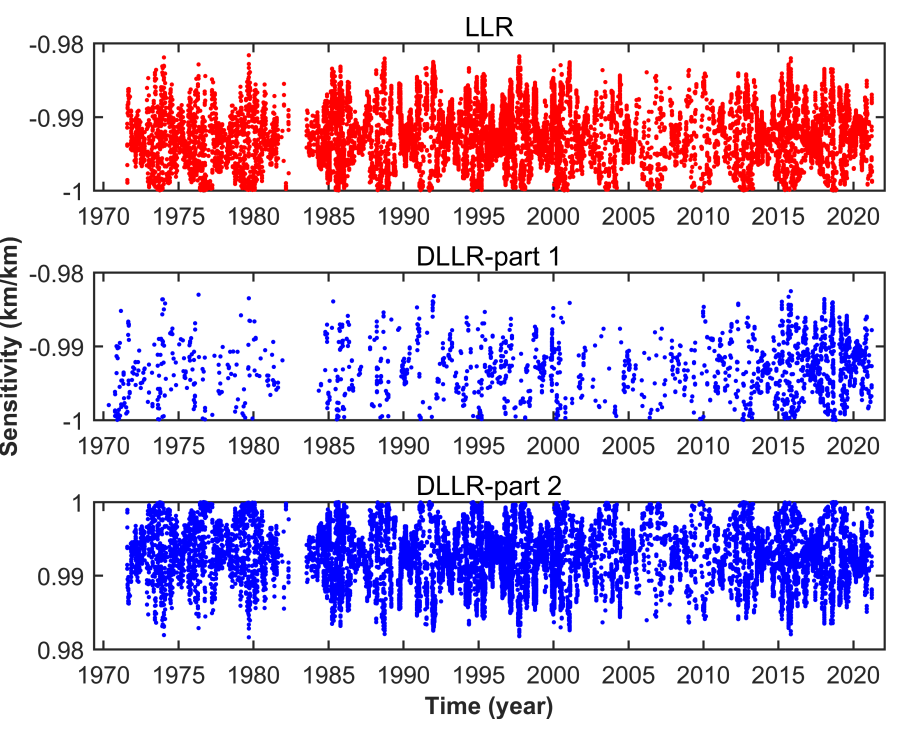

Fig. 10. Sensitivity to $X_{r e f \_A 15}$ for LLR (upper panel) and DLLR negative part (middle panel), positive part (lower panel).

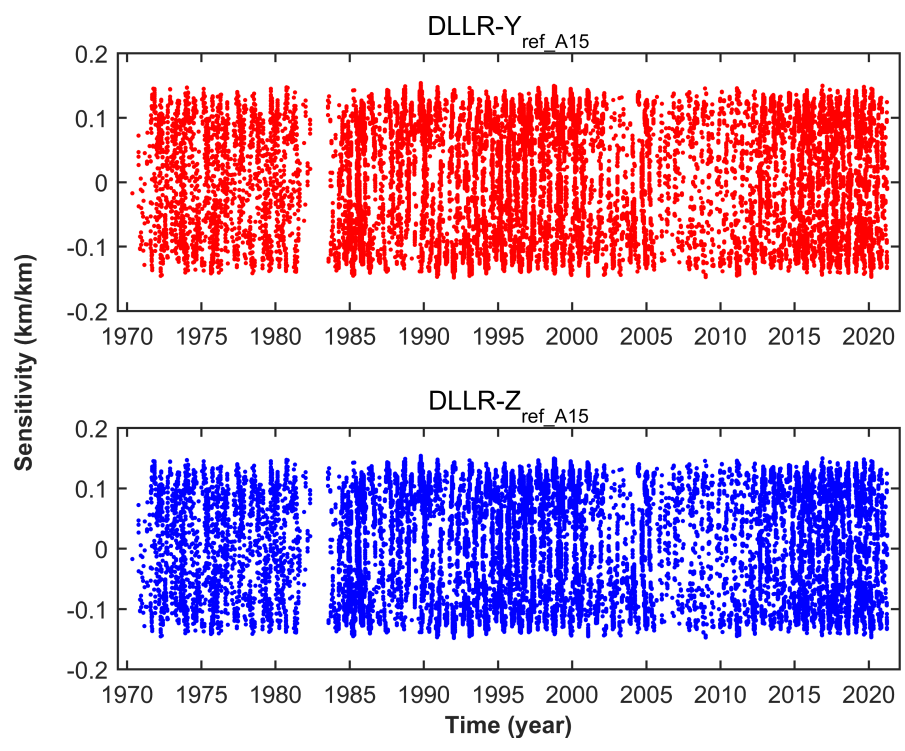

Fig. 11. Sensitivity to $Y_{\text {ref_A15 }}$ (upper panel) and $Z_{\text {ref_A15 }}$ (lower panel) for DLLR.

However, DLLR with a 15-min switching interval is more sensitive to the parameters related to the stations than DLLR with a 1.5-min switching interval. Figures 15-17 show the comparisons of the sensitivities to the OCA station coordinates $\left(X_{\text {sta_OCA }}\right.$, $\left.Y_{\text {sta_OCA }}, Z_{\text {sta_OCA }}\right)$ obtained from DLLR data with 1.5- and 15-min switching intervals. For $X_{\text {sta_OCA }}$ and $Y_{\text {sta_OCA }}$, the magnitude of the sensitivity for the 15-min interval is about five or six times larger than that for the 1.5-min interval. Unlike $X_{\text {sta_OCA }}$ and $Y_{\text {sta_OCA }}$, the sensitivity magnitude of $Z_{\text {sta_oCA }}$ for the 15 -min interval is a bit higher than that for the 1.5 -min interval.

\subsection{Correlations related to stations and reflectors}

The constellation of DLLR is different from that of LLR as a result of including one station and two reflectors in one observation. This motivated us to investigate if it will cause some changes on the correlations among the reflectors and between stations and reflectors. 


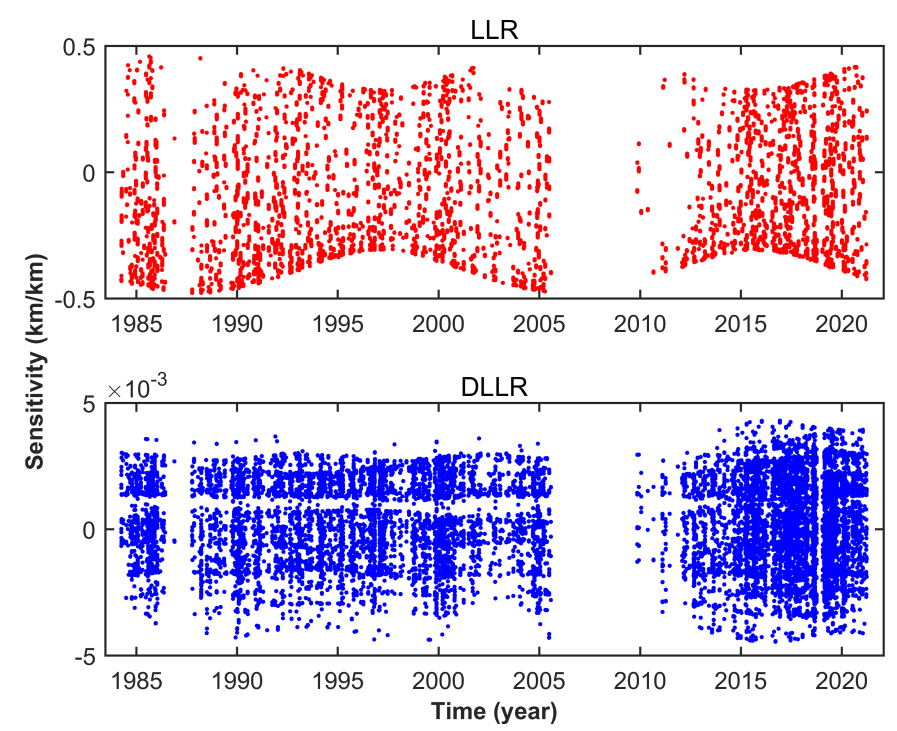

Fig. 12. Sensitivity to $Z_{\text {sta_oCA }}$ for LLR (upper panel) and DLLR (lower panel).

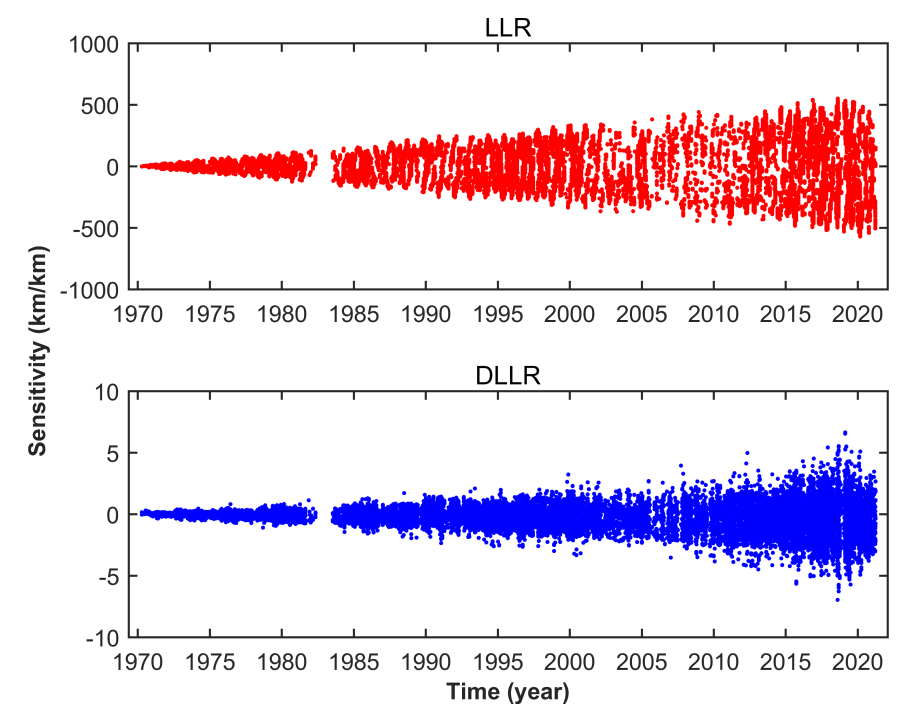

Fig. 13. Sensitivity to $Z_{0}$ for LLR (upper panel) and DLLR (lower panel).

The correlation Cor between one parameter $q$ and another parameter $s$ is calculated via

$\operatorname{Cor}=\frac{\sigma_{q s}}{\sigma_{q} \cdot \sigma_{s}}$

where $\sigma_{q s}$ is the covariance between parameter $q$ and $s$; then $\sigma_{q}$ and $\sigma_{s}$ are the square root of the variances of the parameters $q$ and $s$.

Compared to LLR, DLLR increases the correlations between the $x$-, $y$ - and $z$-coordinates of different reflectors, especially the $z$-coordinate (see Table 3). For DLLR data with 1.5-min as well as for 15 -min switching intervals, these correlations become $100 \%$. This means that we cannot separate the various reflector coordinates in DLLR; and DLLR also increases the correlations between reflectors and stations compared to LLR. The $y$-component of the OCA station coordinates and the $x$-component of the APOLLO coordinates (because OCA and APOLLO are located on different continents) do not show correlations with the $y$-component of the reflectors for LLR. However,

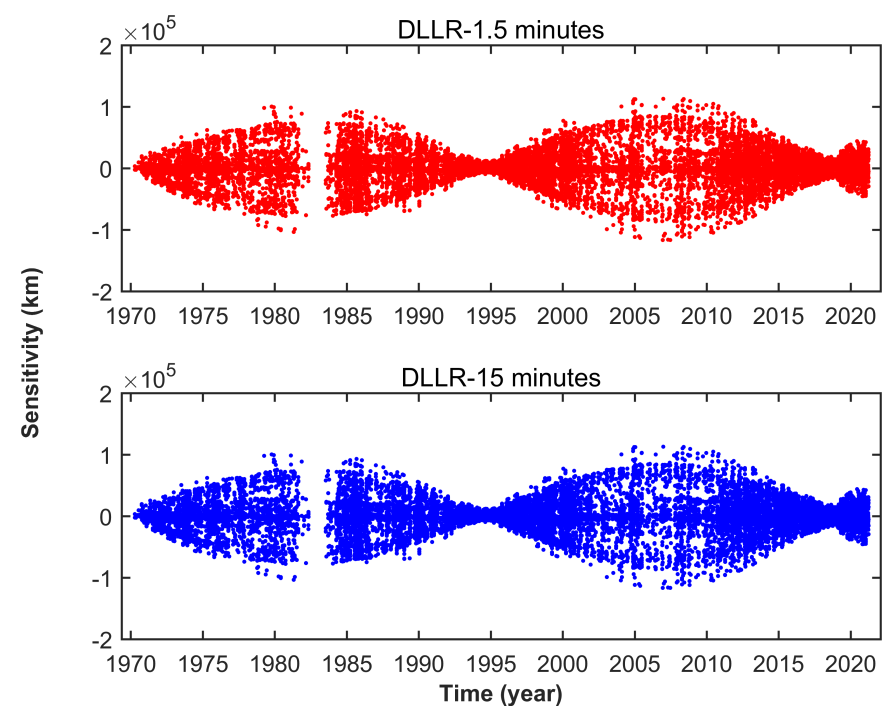

Fig. 14. Sensitivity to $k v c$ for DLLR with a 1.5 -min (upper panel) and 15-min (lower panel) switching interval.

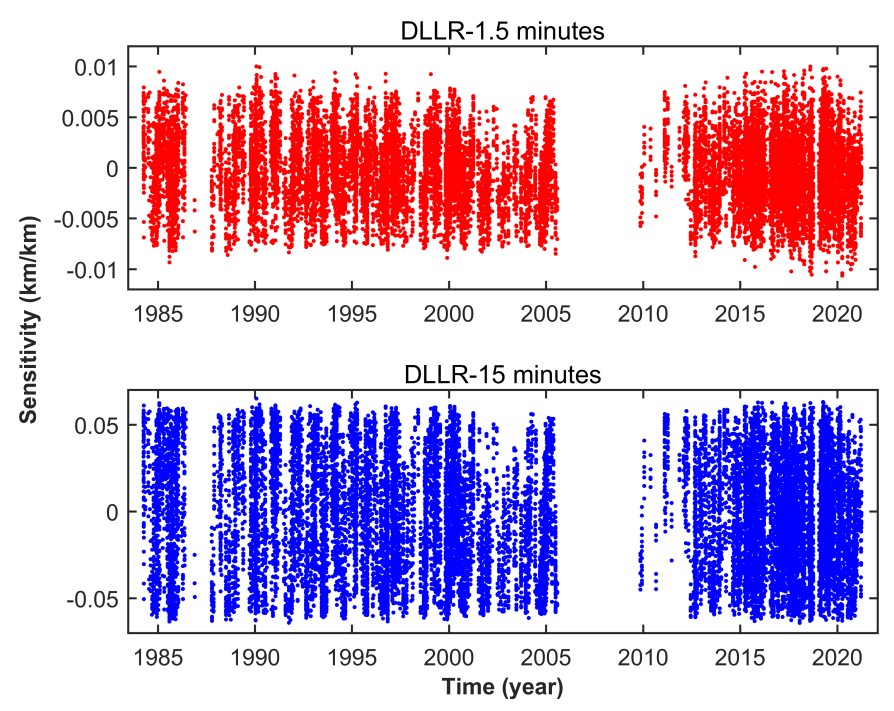

Fig. 15. Sensitivity to $X_{\text {sta_oCA }}$ for DLLR with a 1.5 -min (upper panel) and 15-min (lower panel) switching interval.

for DLLR with a 1.5-min switching interval, the correlation can reach up to $40 \%$, but DLLR data with a 15 -min interval lowers the correlations to $10 \%$.

\subsection{Accuracies of the analyzed parameters}

The accuracies of the estimated parameters have been determined by using the least-squares estimation. Similar parameter accuracies were obtained for DLLR with 1.5-min and 15-min switching intervals. Thus, Table 4 shows the parameter accuracies estimated from LLR and DLLR with a 1.5-min switching interval. For DLLR, the results for assuming a low- and highobservation accuracy are shown.

\subsubsection{Newtonian parameters}

Comparing the accuracies of the parameters from LLR and DLLR with low accuracy (the same as the ranging accuracy of LLR), we divide the parameters into two groups (group I and II). 
Table 3. Comparison of LLR and DLLR for the correlations between the $z$-coordinates of different reflectors.

\begin{tabular}{|c|c|c|c|c|c|}
\hline Reflectors & $\begin{array}{c}\text { Apollo 11 } \\
\text { (LLR/DLLR1.5/DLLR15) }\end{array}$ & $\begin{array}{c}\text { Lunokhod } 2 \\
\text { (LLR/DLLR1.5/DLLR15) }\end{array}$ & $\begin{array}{c}\text { Apollo 14 } \\
\text { (LLR/DLLR1.5/DLLR15) }\end{array}$ & $\begin{array}{c}\text { Apollo 15 } \\
\text { (LLR/DLLR1.5/DLLR15) }\end{array}$ & $\begin{array}{c}\text { Lunokhod } 1 \\
\text { (LLR/DLLR1.5/DLLR15) }\end{array}$ \\
\hline Apollo 11 & $10 / 10 / 10$ & & & & \\
\hline Lunokhod 2 & $4 / 10 / 10$ & $10 / 10 / 10$ & & & \\
\hline Apollo 14 & $6 / 10 / 10$ & $0 / 10 / 10$ & $10 / 10 / 10$ & & \\
\hline Apollo 15 & $4 / 10 / 10$ & $9 / 10 / 10$ & $0 / 10 / 10$ & $10 / 10 / 10$ & \\
\hline Lunokhod 1 & $2 / 10 / 10$ & $9 / 10 / 10$ & $-1 / 10 / 10$ & $9 / 10 / 10$ & $10 / 10 / 10$ \\
\hline
\end{tabular}

Notes. DLLR1.5 and DLLR15 are for DLLR data with a 1.5-min and 15-min switching interval. 10 means that the correlation is $100 \%$.

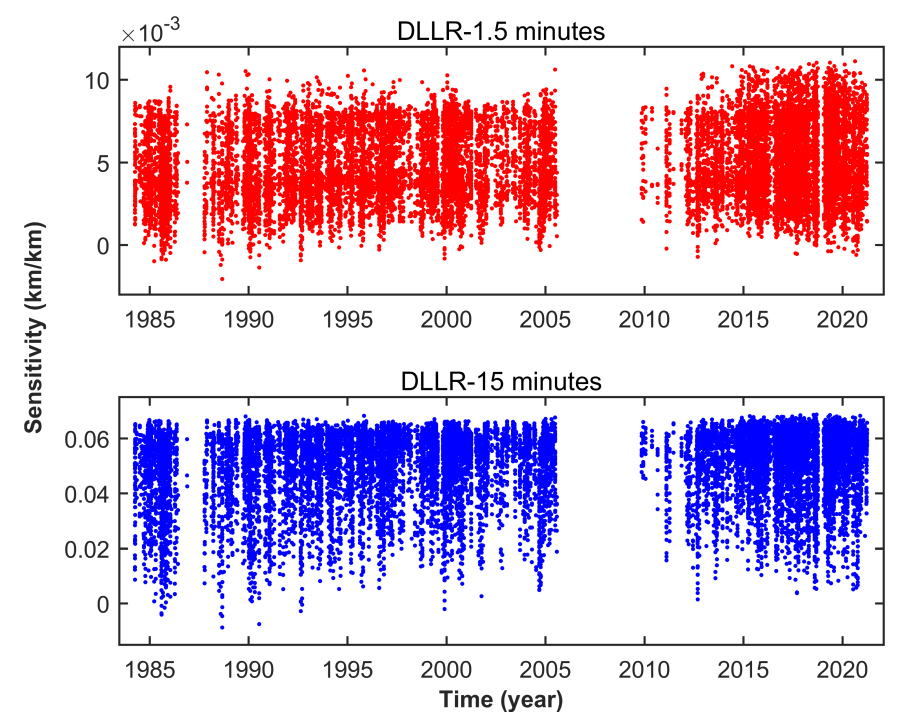

Fig. 16. Sensitivity to $Y_{\text {sta_oCA }}$ for DLLR with a 1.5-min (upper panel) and 15 -min (lower panel) switching interval.

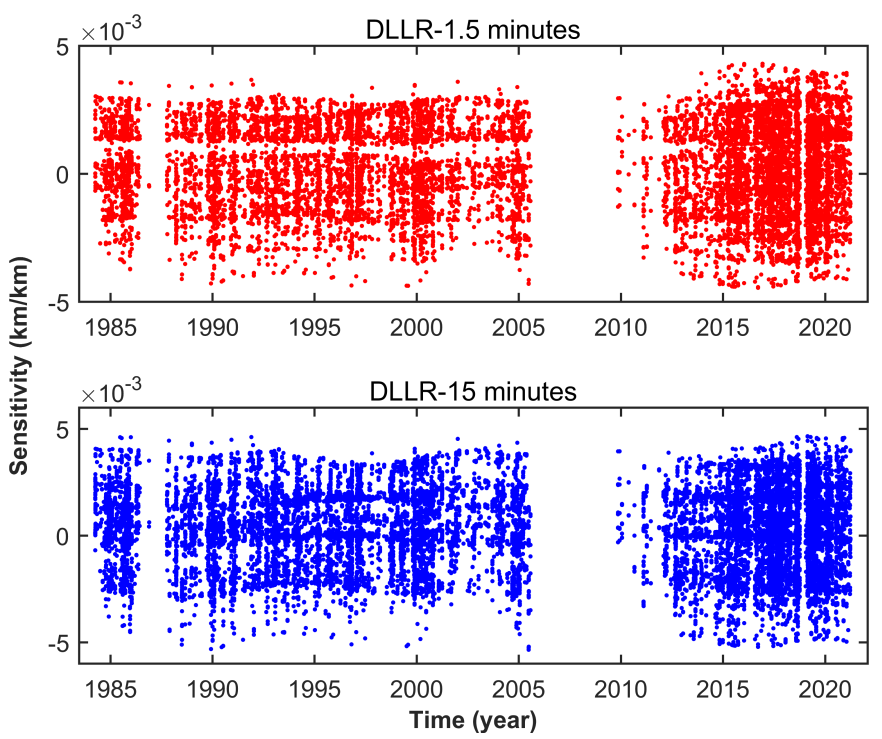

Fig. 17. Sensitivity to $Z_{\text {sta_ocA }}$ for DLLR with a 1.5 -min (upper panel) and 15-min (lower panel) switching interval.

For the parameters of the group I (for example, the initial orientation parameters of the lunar mantle and the oblateness of the lunar core), DLLR reaches the same level of accuracy as standard LLR. That means DLLR can still keep the accuracies of these parameters even with very poor measuring conditions. Almost all parameters included in the group A of the sensitivity part in Sect. 4.1 - LLR and DLLR keep the almost identical sensitivities to the parameters of group A - are in group I (see Table 4), except for the reflector coordinates. LLR and DLLR keep the same sensitivities to the reflector coordinates, but the accuracies calculated by DLLR are much lower than those estimated by LLR, because of the DLLR constellation and the high correlations among the reflectors themselves and between stations and reflectors (see Sect. 4.2). Group II includes the parameters from the group B of the sensitivity part (DLLR is less sensitive than LLR.) and reflector coordinates. The accuracies of these parameters from DLLR under poor measurement conditions are lower than those from LLR. However, this can be compensated if DLLR can reach its expected accuracy (about 200 times improvement compared with LLR ranging accuracy). Then, the accuracies of the parameters in group II estimated from DLLR data with high accuracy are again comparable with those from LLR (see Table 4); however, the accuracies of the parameters in group I are increased largely, that is, two orders of magnitude, by using DLLR data with a high level of accuracy. Furthermore, most parameters in group I are directly related to or have close relation with the lunar interior, for example, $\left(\varphi_{\mathrm{m} 0}\right.$, $\left.\theta_{\mathrm{m} 0}, \psi_{\mathrm{m} 0}\right),\left(\omega_{\mathrm{m} x 0}, \omega_{\mathrm{m} y 0}, \omega_{\mathrm{m} z 0}\right),\left(\omega_{\mathrm{c} x 0}, \omega_{\mathrm{c} y 0}, \omega_{\mathrm{c} z 0}\right), f_{\text {core }}, k v c, C_{22}$, and so on. More details of the relations between these parameters and their relation with the lunar interior can be found in Park et al. (2021) and Williams \& Boggs (2008). It is clear that DLLR is largely beneficial for the exploration of the internal part of the Moon if it can achieve the envisaged accuracy in the future. This remains one of the main goals of DLLR.

\subsubsection{EP parameters}

Apart from studying the Newtonian parameters, we also estimated the parameters related to the EP tests in the gravity field of the galactic dark matter (assumed in the galaxy's center) and the Sun by using LLR and DLLR data with high accuracy. If the EP is violated, a difference between the ratio $\frac{m_{\mathrm{g}}}{m_{i}}$ for the Moon and Earth in the gravity field of the Sun $\delta\left(\frac{m_{\mathrm{g}}}{m_{i}}\right)_{\mathrm{EM}}$ (Biskupek et al. 2021) and the additional acceleration of the Moon relative to the Earth towards the galactic center $\delta a_{\mathrm{dm}}$ (Zhang et al. 2020) would be obtained. Estimating these two effects using LLR, we get $\delta a_{\mathrm{dm}}=(2.3 \pm 4.1) \times 10^{-17} \mathrm{~m} \mathrm{~s}^{-2}$, and $\delta\left(\frac{m_{\mathrm{g}}}{m_{i}}\right)_{\mathrm{EM}}=$ $(-0.6 \pm 2.1) \times 10^{-14}$. Applying DLLR, one can improve the estimation accuracy of $\delta a_{\mathrm{dm}}$ about three-fold and $\delta\left(\frac{m_{\mathrm{g}}}{m_{i}}\right)_{\mathrm{EM}}$ about 2.5-fold.

\section{Summary and outlook}

Differential Lunar Laser Ranging (DLLR) is a promising technique meant to significantly enhance our knowledge of the lunar 
Table 4. Comparison of DLLR and LLR with respect to the accuracies of the estimated parameters.

\begin{tabular}{|c|c|c|c|c|}
\hline Group & Parameter & LLR & $\begin{array}{c}\text { DLLR } \\
(1.5 \mathrm{~min}, \text { low accuracy) } \\
\end{array}$ & $\begin{array}{c}\text { DLLR } \\
\text { (1.5 min, high accuracy) }\end{array}$ \\
\hline \multirow{12}{*}{ Group I } & $\varphi_{\mathrm{m} 0}[\mathrm{rad}]$ & $0.9 \times 10^{-8}$ & $1.1 \times 10^{-8}$ & $0.6 \times 10^{-10}$ \\
\hline & $\theta_{\mathrm{m} 0}$ & $4.4 \times 10^{-9}$ & $2.7 \times 10^{-9}$ & $1.4 \times 10^{-11}$ \\
\hline & $\psi_{\mathrm{m} 0}$ & $1.5 \times 10^{-8}$ & $2.1 \times 10^{-8}$ & $1.0 \times 10^{-10}$ \\
\hline & $\omega_{\mathrm{m} x 0}\left[\mathrm{rad} \mathrm{s}^{-1}\right]$ & $7.8 \times 10^{-15}$ & $4.3 \times 10^{-15}$ & $2.2 \times 10^{-17}$ \\
\hline & $\omega_{\mathrm{m} y 0}$ & $5.1 \times 10^{-15}$ & $5.8 \times 10^{-15}$ & $2.9 \times 10^{-17}$ \\
\hline & $\omega_{\mathrm{m} z 0}$ & $2.1 \times 10^{-16}$ & $1.2 \times 10^{-16}$ & $0.6 \times 10^{-18}$ \\
\hline & $\omega_{\mathrm{c} x 0}$ & $2.6 \times 10^{-11}$ & $2.0 \times 10^{-11}$ & $1.0 \times 10^{-13}$ \\
\hline & $\omega_{\mathrm{c} y 0}$ & $2.1 \times 10^{-11}$ & $1.8 \times 10^{-11}$ & $0.9 \times 10^{-13}$ \\
\hline & $\omega_{\mathrm{c} z 0}$ & $1.6 \times 10^{-10}$ & $3.4 \times 10^{-10}$ & $1.7 \times 10^{-12}$ \\
\hline & $C_{22}$ [unitless] & $3.6 \times 10^{-11}$ & $1.8 \times 10^{-11}$ & $0.9 \times 10^{-13}$ \\
\hline & $f_{\text {core }}$ & $1.6 \times 10^{-6}$ & $1.1 \times 10^{-6}$ & $0.6 \times 10^{-8}$ \\
\hline & $k v c$ & $1.1 \times 10^{-10}$ & $1.5 \times 10^{-10}$ & $0.8 \times 10^{-12}$ \\
\hline \multirow{12}{*}{ Group II } & $X_{0}[\mathrm{~km}]$ & $8.5 \times 10^{-5}$ & $4.6 \times 10^{-3}$ & $2.3 \times 10^{-5}$ \\
\hline & $Y_{0}$ & $3.0 \times 10^{-5}$ & $3.8 \times 10^{-3}$ & $1.9 \times 10^{-5}$ \\
\hline & $Z_{0}$ & $2.9 \times 10^{-5}$ & $2.2 \times 10^{-3}$ & $1.1 \times 10^{-5}$ \\
\hline & $V_{x 0}\left[\mathrm{~km} \mathrm{~s}^{-1}\right]$ & $1.0 \times 10^{-10}$ & $1.3 \times 10^{-8}$ & $0.6 \times 10^{-10}$ \\
\hline & $V_{y 0}$ & $2.1 \times 10^{-10}$ & $1.1 \times 10^{-8}$ & $0.6 \times 10^{-10}$ \\
\hline & $V_{z 0}$ & $1.3 \times 10^{-10}$ & $0.7 \times 10^{-8}$ & $0.3 \times 10^{-10}$ \\
\hline & $X_{r e f \_A 15}[\mathrm{~km}]$ & $2.4 \times 10^{-5}$ & $6.7 \times 10^{-3}$ & $3.4 \times 10^{-5}$ \\
\hline & $Y_{\text {ref_A } A 15}$ & $1.9 \times 10^{-5}$ & $3.7 \times 10^{-3}$ & $1.8 \times 10^{-5}$ \\
\hline & $Z_{r e f \_A 15}$ & $1.4 \times 10^{-5}$ & $1.2 \times 10^{-3}$ & $0.6 \times 10^{-5}$ \\
\hline & $X_{\text {sta_OCA }}[\mathrm{km}]$ & $1.1 \times 10^{-6}$ & $0.7 \times 10^{-4}$ & $0.4 \times 10^{-6}$ \\
\hline & $Y_{\text {sta_OCA }}$ & $7.9 \times 10^{-7}$ & $5.7 \times 10^{-5}$ & $2.9 \times 10^{-7}$ \\
\hline & $Z_{\text {Sta_OCA }}$ & $3.4 \times 10^{-6}$ & $3.2 \times 10^{-4}$ & $1.6 \times 10^{-6}$ \\
\hline
\end{tabular}

interior and beneficial in relativity tests. By applying a differential mode between several reflectors, it can largely reduce the Earth's atmospheric error, which is the main error source of LLR. As a result, its expected accuracy is about 200 times better than LLR. We simulated DLLR data to investigate the characteristics of DLLR by comparing them with LLR. We find that DLLR can keep the sensitivity for some parameters of the lunar part, especially the parameters relevant to the lunar interior, for example, the rotation parameters of the Moon and the oblateness of the lunar core. Additionally, DLLR can greatly improve the estimation accuracies of these parameters compared to the results from LLR. This supports its main goal to achieve a more comprehensive understanding of the lunar interior. Even if the measurement conditions are not good (if the accuracies of DLLR observations are low), DLLR can still keep the accuracies of these parameters at a similar level as LLR. Reflector coordinates are a special case in DLLR. Because of the DLLR constellation, they can hardly be improved by highly accurate DLLR data, although DLLR has the same sensitivity to them as LLR. Compared with LLR, we can see that DLLR has a lower sensitivity to the parameters of the station part, such as station coordinates, due to its cancelling effect resulting from its differential measuring mode. However, this is not expected to cause a problem, as long as DLLR reaches its high level of accuracy. In that case, DLLR can get a comparable accuracy with regard to LLR. The correlations among the reflectors themselves and between stations and reflectors increased. Switching intervals of DLLR data plays a role for these correlations. Large switching intervals (e.g., $15 \mathrm{~min}$ ) are helpful for the decorrelation of station and reflector coordinates. Moreover, a large switching interval is more beneficial to station coordinates than a small one. In this paper, we also show that the accuracy of the EP test (as one of many relativity tests) can be improved by DLLR.

We will continue to study DLLR also for more relativity tests, for example, examining the time variation of the gravitational constant. The combination of LLR and DLLR data will also be a key point in our future analysis. Furthermore, when more novel types of reflectors are placed on the Moon, it is expected that DLLR and LLR estimation will be further enhanced. We will then investigate their benefits. For the lunar reflectors, we also expect some improvements in the differential reflector coordinates when using DLLR. However, this still needs further investigation in the future.

Acknowledgements. Current LLR data were collected, archived, and distributed under the auspices of the International Laser Ranging Service (ILRS) (Pearlman et al. 2019). We acknowledge with thanks that since 1969 LLR data has been obtained under the efforts of the personnel at the McDonald Observatory in Texas, USA, the LURE Observatory in Maui, Hawaii, USA, the Observatoire de la Côte dAzur in France, the Wettzell Laser Ranging System in Germany, the Matera Laser Ranging station in Italy and the Apache Point Observatory in New Mexico, USA. This research was funded by the Deutsche Forschungsgemeinschaft (DFG, German Research Foundation) under Germany's Excellence Strategy-EXC-2123 QuantumFrontiers-390837967. The study brought together the efforts of all authors. Everyone provided good suggestions. The first draft of the manuscript was written by MZ. All authors made comments on previous versions of the manuscript. The final version was approved by all authors.

\section{References}

Biskupek, L. 2015, PhD thesis, Fachrichtung Geodäsie und Geoinformatik der Leibniz Universität Hannover

Biskupek, L., Müller, J., \& Torre, J.-M. 2021, Universe, 7, 34

Chapront, J., \& Francou, G. 2006, Lunar Laser Ranging: Measurements, Analysis and Contribution to the Reference Systems (Citeseer) 
Ciocci, E., Martini, M., Contessa, S., et al. 2017, Adv. Space Res., 60, 1300

Courde, C., Torre, J., Samain, E., et al. 2017, A\&A, 602, A90

Currie, D., Dell'Agnello, S., \& Delle Monache, G. 2011, Acta Astronautica, 68, 667

Dehant, V., Park, R., Dirkx, D., et al. 2017, Space Sci. Rev., 212, 1433

Einstein, A., Infeld, L., \& Hoffmann, B. 1938, Ann. Math., 65

Garattini, M., Dell'Agnello, S., Currie, D., et al. 2013, Acta Polytech., 53, 821

Garcia, R. F., Khan, A., Drilleau, M., et al. 2019, Space Sci. Rev., 215, 1

Hofmann, F. 2017, PhD thesis, Fachrichtung Geodäsie und Geoinformatik der Leibniz Universität Hannover

Hofmann, F., \& Müller, J. 2018, Class. Quant. Grav., 35, 035015

Hofmann, F., Biskupek, L., \& Müller, J. 2018, J. Geodesy, 92, 975

Mendes, V., \& Pavlis, E. C. 2004, Geophys. Res. Lett., 31

Mendes, V., Prates, G., Pavlis, E. C., Pavlis, D., \& Langley, R. 2002, Geophys. Res. Lett., 29, 53

Moyer, T. D. 1971, Mathematical formulation of the double precision orbit determination program/dpodp, Tech. rep.

Müller, J., Biskupek, L., Hofmann, F., \& Mai, E. 2014, in Volume 2: Applications and Experiments (de Gruyter), 103

Müller, J., Murphy, T. W., Schreiber, U., et al. 2019, J. Geodesy, 93, 2195

Murphy, T. 2013, Rep. Progr. Phys., 76, 076901

Murphy, T., Adelberger, E. G., Battat, J., et al. 2008, PASP, 120, 20

Noll, C. E. 2010, Adv. Space Res., 45, 1421

Park, R. S., Folkner, W. M., Williams, J. G., \& Boggs, D. H. 2021, Astron. J., 161,105

Pearlman, M. R., Noll, C. E., Pavlis, E. C., et al. 2019, J. Geodesy, 93, 2161

Petit, G., \& Luzum, B. 2010, IERS conventions 2010, Tech. rep., Bureau International des Poids et mesures sevres (France)
Schreiber, K. U., Eckl, J. J., Leidig, A., et al. 2019, in AGU Fall Meeting Abstracts, 2019, G31B-0647

Shapiro, I. I. 1964, Phys. Rev. Lett., 13, 789

Singh, V. V., Biskupek, L., Müller, J., \& Zhang, M. 2021, ArXiv e-prints [arXiv:2110.14274]

Turyshev, S. G., Williams, J. G., Folkner, W. M., et al. 2013, Exp. Astron., 36, 105

Turyshev, S. G., Shao, M., Hanh, I., Williams, J. G., \& Trahan, R. 2018, in Proceedings of the 21st International Workshop on Laser Ranging, Canberra, Australia, 5

Viswanathan, V., Fienga, A., Minazzoli, O., et al. 2018, MNRAS, 476, 1877

Will, C. M. 2018, Theory and Experiment in Gravitational Physics (Cambridge University Press)

Williams, J. G., \& Boggs, D. H. 2008, in Proceedings of the 16th International Workshop on Laser Ranging, Poznan, Poland, 1317

Williams, J. G., Turyshev, S. G., \& Murphy Jr, T. W. 2004, Int. J. Mod. Phys. D, 13,567

Williams, J. G., Turyshev, S. G., \& Boggs, D. H. 2009, Int. J. Mod. Phys. D, 18, 1129

Williams, J. G., Turyshev, S. G., \& Boggs, D. H. 2012, Class. Quant. Grav., 29, 184004

Williams, J. G., Konopliv, A. S., Boggs, D. H., et al. 2014, J. Geophys. Res.: Planets, 119, 1546

Zhang, M. 2022, Dataset: Simulated DLLR Data for Timespan 1970-2021, Research Data Repository of the Leibniz University Hannover, https:// doi.org/10.25835/hiioueb9

Zhang, M., Müller, J., \& Biskupek, L. 2020, Celes. Mech. Dyn. Astron., 132 